\title{
A Global Operator Formalism on Higher Genus Riemann Surfaces: $b-c$ Systems
}

\author{
Loriano Bonora ${ }^{1,2}$, Adrian Lugo $^{1}$, Marco Matone ${ }^{1,2}$, and Jorge Russo ${ }^{1}$ \\ ${ }^{1}$ I.S.A.S./S.I.S.S.A.-Strada Costiera 11, Trieste, Italy \\ ${ }^{2}$ I.N.F.N., Sezione di Trieste, Italy
}

\begin{abstract}
We explicitly construct bases for meromorphic $\lambda$-differentials over genus $g$ Riemann surfaces. With the help of these bases we introduce a new operator formalism over Riemann surfaces which closely resembles the operator formalism on the sphere. As an application we calculate the propagators for $b-c$ systems with arbitrary integer or half-integer $\lambda$ (in the Ramond and Neveu-Schwarz sectors). We also give explicit expressions for the zero modes and for the Teichmüller deformations for a generic Riemann surface.
\end{abstract}

\section{Introduction}

Operator formulations of conformal field theories over a generic Riemann surface $\Sigma$, as opposed to the path integral formulation, have recently been the object of intensive research [1-7]. The common feature of these approaches is that they privilege the local description of conformal field theories over a disk cut out from the Riemann surface. The globalization is essentially obtained via Bogoliubov transformations relating states over the disk to states over the Riemann surface without disk. This formulation finds its natural mathematical framework in the Grassmannian formalism developed in $[8,9]$. In a different mathematical context, we also recall the important related results of $[10,11]$ on the action of the Virasoro algebra on the moduli space. Needless to say, a conformal field theory formulated in this way over a non-trivial (non-spherical) topology looks rather involved. We think a simpler and clearer formalism is now at hand, due to the work of Krichever and Novikov (KN) $[12,13]$, who recently suggested new bases for meromorphic tensor fields on genus $g$ Riemann surfaces which are holomorphic outside two points $P_{+}$and $P_{-}$. These bases are uniquely determined up to numerical constants. It is therefore possible to closely mimic a conformal field theory over a sphere $[14,15]$ (where $P_{ \pm}$are identified with the North and South poles), the KN bases playing the role of the monomials $z^{n}(n \in Z)$ over the sphere. In other words the $\mathrm{KN}$ bases provide a mean to globally Laurent-like expand any tensor field of 
the type described before on genus $g$ Riemann surfaces. This fact lends itself to an operator formalism interpretation similar to the analog over the sphere. As in the latter case, the coefficients of the above expansions will be interpreted as creation and annihilation operators acting on a suitable "vacuum" $|0\rangle_{\Sigma}$ characteristic of the given Riemann Surface $\Sigma$ of genus $g$. The corresponding excitations do not have, of course, the same physical meaning as the analogs on the sphere, but are in general genus dependent combinations of the latter.

A conformal field theory based on the $\mathrm{KN}$ bases has already been partially developed in $[16,17,18]$. In [16] it was shown that one can construct for a bosonic string theory over any $\Sigma$ a BRST charge which becomes nilpotent in $D=26$. This was extended to the superstring case in [17], while in [18] it was shown that the Sugawara construction can be carried out on any Riemann surface.

In this paper we start developing systematically the operator formalism over a generic $\Sigma$ by means of the $\mathrm{KN}$ bases, by considering the simplest cases of conformal field theories: the $b-c$ chiral systems. Throughout this paper $b$ and $c$ are tensors of weights $\lambda$ and $1-\lambda$ respectively; they may be commuting or anticommuting fields. In string theory the anticommuting system with $\lambda=2$ is of special interest because it corresponds to reparametrization ghosts. In superstring theory we have in addition matter with $\lambda=1 / 2$ and the superghost system with $\lambda=3 / 2$. Our aim is to be able to compute any correlation function for these systems. To this end we need an explicit expression for the KN bases: we provide it in Sect. I. In particular we write a basis for the zero modes of arbitrary $\lambda$-differentials. Next (Sect. II), we discuss the Laurent-like expansions of these fields, the operator interpretation of the coefficients and the vacuum state, as well as their relation to the corresponding genus zero quantities. In Sect. III we compute the Szegö kernel for an arbitrary $b-c$ system, that is, the relevant $b-c$ system for any genus and any integer or halfinteger $\lambda$ in both Neveu-Schwarz and Ramond cases. In Sect. IV we discuss zero modes, Teichmüller deformations and the relation with the path integral approach. In Appendices A and B we set the notation and recall some basic facts about theta functions, theta divisors, and spin structures.

\section{Krichever-Novikov Bases. Explicit Construction}

Let $\Sigma$ be a compact Riemann surface of genus $g$ and $P_{+}, P_{-}$two distinguished points in general position.

The KN bases $[12,13]$ are bases for the spaces of meromorphic tensors of weight $\lambda$ on the Riemann surface, which are holomorphic outside $P_{+}$and $P_{-}$.

\section{Case of Integer $\lambda$}

For integer $\lambda \neq 0,1$ and $g>1$, the Riemann-Roch theorem guarantees the existence and uniqueness (up to a multiplicative constant) of tensors of conformal weight $\lambda$ which in a neighborhood of $P_{+}$and $P_{-}$have the following behaviour:

$$
f_{j}^{(\lambda)}\left(z_{ \pm}\right)=\varphi_{j}^{(\lambda) \pm} z_{ \pm}^{ \pm j-s(\lambda)}\left(1+O\left(z_{ \pm}\right)\right)\left(d z_{ \pm}\right)^{\lambda},
$$


where

$$
s(\lambda)=g / 2-\lambda(g-1) .
$$

Here $z_{ \pm}\left(P_{ \pm}\right)=0$, where $z_{ \pm}$are local coordinates at $P_{ \pm}$. The multiplicative constant

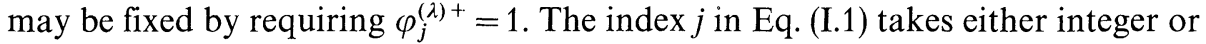
half-integer values depending on whether $g$ is even or odd, respectively. When $j=s(\lambda), \ldots,-s(\lambda)$ and $\lambda>1$ we have a basis for zero modes of the $\bar{\partial}$-operator acting on $\lambda$-differentials.

Note that uniqueness can also be proved from the Noether "gap" theorem [19]. In fact, let us assume there are two sections $f_{j}^{(\lambda)}$ and $f_{j}^{\prime(\lambda)}$ of $K^{\lambda}$ satisfying (I.1) and define a function $h$ as the quotient of them. Since $f_{j}^{(\lambda)}$ and $f_{j}^{\prime(\lambda)}$ have $g$ zeroes outside $P_{ \pm}$, it follows that $h$ is a meromorphic function with a number of poles between zero and $g$. But the Noether "gap" theorem states that there exist no functions with a number of poles in general position between 1 and $g$. This implies that $h$ is necessarily holomorphic and therefore a constant.

For $\lambda=0$ the behaviour is modified with respect to Eq. (I.1). Let $A_{j},|j| \geqq g / 2+1$, be the unique function which in a neighborhood of $P_{ \pm}$has the Laurent expansion

$$
A_{j}\left(z_{ \pm}\right)=\alpha_{j}^{ \pm} z_{ \pm}^{ \pm j-g / 2}\left(1+O\left(z_{ \pm}\right)\right) .
$$

(As before, $j$ is integer or half-integer depending on the parity of $g$ ). For $j=-g / 2, \ldots, g / 2-1$ we take the functions with the following behaviour:

$$
A_{j}\left(z_{ \pm}\right)=\alpha_{j}^{ \pm} z_{ \pm}^{ \pm j-g / 2_{-i}^{+0}}\left(1+O\left(z_{ \pm}\right)\right) .
$$

These conditions define $A_{j}$ uniquely up to addition of a constant. For $j=g / 2$ we choose $A_{g / 2}=1$; this completes the basis of meromorphic functions.

For $\lambda=1$, we take the basis of one-forms as follows: in the range $|j| \geqq g / 2+1$, $\omega^{j}=f_{-j}^{(1)}$, with $f_{-j}^{(1)}$ given by (I.1); for $j=-g / 2, \ldots, g / 2-1$, those specified by the local series

$$
\omega^{j}\left(z_{ \pm}\right)=\beta_{j}^{ \pm} z_{ \pm}^{\mp j+g / 2_{+0}^{-1}}\left(1+O\left(z_{ \pm}\right)\right) d z_{ \pm} .
$$

Finally, we take $\omega^{g / 2}$ as the Abelian differential of the third kind with simple poles in $P_{ \pm}$and residues \pm 1 , normalized in such a way that its periods over all cycles be purely imaginary.

In the $g=1$ case, the number of zeros is equal to the number of poles for any section of $K^{\lambda}$, for any $\lambda$; the existence of a holomorphic (and therefore without zeroes and poles) one-form $\eta$ enables us to write the $\mathrm{KN}$ bases as follows:

$$
f_{j}^{(\lambda)}=A_{j} \eta^{\lambda}
$$

where the $A_{j}$ 's are defined as before.

Let us now move to the explicit construction of these bases (in what follows, we will make extensive use of definitions and properties given in Appendix A). Looking at (I.1), we observe that this behaviour is correctly reproduced by using prime forms as follows:

$$
f_{j}^{(\lambda)} \approx E\left(P, P_{+}\right)^{j-s(\lambda)} / E\left(P, P_{-}\right)^{j+s(\lambda)} .
$$

The correct weight in the $P$-variable is obtained by means of the $\sigma$-differential

$$
f_{j}^{(\lambda)} \approx\left(E\left(P, P_{+}\right)^{j-s(\lambda)} / E\left(P, P_{-}\right)^{j+s(\lambda)}\right) \sigma(P)^{2 \lambda-1} .
$$


Finally, we require $f_{j}^{(\lambda)}$ to be single-valued. To this purpose we introduce a $\theta$-function

$$
f_{j}^{(\lambda)}(P)=N_{j}^{(\lambda)}\left(P_{+}, P_{-}\right) \frac{E\left(P, P_{+}\right)^{j-s(\lambda)}}{E\left(P, P_{-}\right)^{j+s(\lambda)}} \sigma(P)^{2 \lambda-1} \theta(P+e(\lambda, j)),
$$

where

$$
e(\lambda, j)=(j-s(\lambda)) P_{+}-(j+s(\lambda)) P_{-}+(1-2 \lambda) \Delta,
$$

and

$$
N_{j}^{(\lambda)}\left(P_{+}, P_{-}\right)=\frac{E\left(P_{+}, P_{-}\right)^{j+s(\lambda)} \sigma\left(P_{+}\right)^{1-2 \lambda} h\left(P_{+}\right)^{2(j-s(\lambda)+\lambda)}}{\theta\left(P_{+}+e(\lambda, j)\right)} .
$$

Note that the $\theta$-function gives the $g$ zeroes of $f_{j}^{(\lambda)}$ outside $P_{ \pm}$; the constant $N_{j}^{(\lambda)}\left(P_{+}, P_{-}\right)$is chosen in order to satisfy a duality condition to be defined below. ${ }^{1}$

For $g=1$ or $\lambda=0,1$, Eq. (I.6) does not work in the interval $-g / 2 \leqq j \leqq g / 2$. In fact, in these cases the $\theta$-function has zeroes in $P_{+}$and $P_{-}$which cancel the poles of the prime forms, as it is easy to verify by using the Riemann vanishing theorem and the relation $\Theta+2 \gamma_{(\alpha \beta)}=\Theta$, where $\Theta$ is the locus of zeroes of the $\theta$-function and $\gamma_{(\alpha \beta)}$ depends on the Riemann class and on the spin structure (see Appendix B).

For $\lambda=0$ the expressions for any $g$ are defined by $|j|>g / 2$ :

$$
A_{j}(P)=\text { as (I.6) with } \lambda=0 \text {, }
$$

$-g / 2 \leqq j \leqq g / 2-1:$

$$
\begin{aligned}
A_{j}(P)= & N_{j}^{(0)}\left(P_{ \pm}, P_{g+1}\right) \frac{E\left(P, P_{+}\right)^{j-g / 2} E\left(P, P_{g+1}\right)}{E\left(P, P_{-}\right)^{j+g / 2+1}} \\
& \times \sigma(P)^{-1} \theta(P+e(j))-a_{j}\left(P_{ \pm}, P_{g+1}\right)-a_{j}\left(P_{ \pm}, P_{g+1}\right),
\end{aligned}
$$

where

$$
\begin{aligned}
& e(j)=(j-g / 2) P_{+}-(j+g / 2+1) P_{-}+P_{g+1}+\Delta, \\
& a_{j}\left(P_{ \pm}, P_{g+1}\right)=\frac{1}{2 \pi i} \oint_{C}\left(A_{j}(P)+a_{j}\left(P_{ \pm}, P_{g+1}\right)\right) \omega_{g / 2},
\end{aligned}
$$

and

$$
N_{j}^{(0)}\left(P_{ \pm}, P_{g+1}\right)=\frac{E\left(P_{+}, P_{-}\right)^{j+g / 2+1} \sigma\left(P_{+}\right) h\left(P_{+}\right)^{2(j-g / 2)}}{\theta\left(P_{+}+e(j)\right) E\left(P_{+}, P_{g+1}\right)} .
$$

Here $P_{g+1}$ is an arbitrary point different from $P_{ \pm}$[as we have already said, the $A_{j}$ 's defined by (I.3) are fixed up to addition of a constant; this arbitrariness is reflected in (I.7) through the point $\left.P_{q+1}\right], \omega^{g / 2}$ is given below in Eq. (I.9), and $C$ is any contour which separates $P_{+}$and $P_{-}$). Finally we take $A_{g / 2}=1$.

\footnotetext{
${ }^{1}$ One can check that this choice of the coefficient $N_{J}^{(\lambda)}$ makes $f_{j}^{(\lambda)}$ single-valued and with the right weight in both $P_{+}$and $P_{-}$variables
} 
For $\lambda=1$ the elements of the basis of one-forms take the following form:

$|j|>g / 2$

$$
\omega^{j}(P)=f_{-j}^{(1)}, \quad \text { according to (I.6) }
$$

$-g / 2 \leqq j \leqq g / 2-1$

$$
\omega^{j}(P)=N_{-j}^{(1)}\left(P_{ \pm}, P_{g+1}\right) \frac{E\left(P, P_{-}\right)^{j+g / 2} \sigma(P)}{E\left(P, P_{+}\right)^{j-g / 2+1} E\left(P, P_{g+1}\right)} \theta(P+e(j)),
$$

where

$$
e(j)=(j+g / 2) P_{-}-(j-g / 2+1) P_{+}-P_{g+1}-\Delta,
$$

and

$$
N_{-j}^{(1)}\left(P_{ \pm}, P_{g+1}\right)=\frac{E\left(P_{+}, P_{-}\right)^{-j-g / 2} E\left(P_{+}, P_{g+1}\right) h\left(P_{+}\right)^{-2(j-g / 2)}}{\theta\left(P_{+}+e(j)\right) \sigma\left(P_{+}\right)}
$$

$j=g / 2$

$$
\omega^{g / 2}(P)=d\left[\ln \left(E\left(P, P_{+}\right) / E\left(P, P_{-}\right)\right)\right]-2 \pi i \sum_{i, j=1}^{g} \operatorname{Im}\left(\int_{P_{-}}^{P_{+}} \eta_{i}\right)(\operatorname{Im} \Omega)_{i j}^{-1} \eta_{j}(P),
$$

where $\eta_{j}$ are the $g$ holomorphic differentials with the standard normalization (see Appendix A). ${ }^{2}$

In the genus one case, considering Eq. (I.5) we can define the following expressions:

for $|j|>1 / 2$

$$
f_{j}^{(\lambda)}(P)=N_{j}^{(\lambda)}\left(P_{+}, P_{-}\right) \frac{E\left(P, P_{+}\right)^{j-1 / 2}}{E\left(P, P_{-}\right)^{j+1 / 2}} \sigma(P)^{2 \lambda-1} \theta(P+e(j)),
$$

where

$$
\begin{gathered}
e(j)=(j-1 / 2) P_{+}-(j+1 / 2) P_{-}+\Delta \\
N_{j}^{(\lambda)}\left(P_{+}, P_{-}\right)=\frac{E\left(P_{+}, P_{-}\right)^{j+1 / 2} \sigma\left(P_{+}\right)^{2 j}}{\theta\left(P_{+}+e(j)\right)}
\end{gathered}
$$

for $|j|=1 / 2$

$$
f_{1 / 2}^{(\lambda)}(P)=\sigma(P)^{2 \lambda}
$$

and

$$
\begin{aligned}
f_{-1 / 2}^{(\lambda)}(P)= & N_{-1 / 2}^{(\lambda)}\left(P_{ \pm}, P_{2}\right) \frac{E\left(P, P_{2}\right) \sigma(P)^{2 \lambda-1}}{E\left(P, P_{+}\right) E\left(P, P_{-}\right)} \theta(P-e) \\
& -c\left(P_{ \pm}, P_{2}\right) f_{1 / 2}^{(\lambda)}(P),
\end{aligned}
$$

\footnotetext{
${ }^{2}$ Note that in Eq. (I.8) we have no pole in $P_{g+1}$ because the $\theta$-function has a zero there which cancels the zero of $E\left(P, P_{g+1}\right)$ for any $j=-g / 2, \ldots, g / 2-1$
} 
where $e=P_{+}+P_{-}-P_{2}-\Delta$, and

$$
c\left(P_{ \pm}, P_{2}\right)=\left(\frac{E\left(P_{+}, P_{-}\right) \sigma\left(P_{+}\right)^{-1}}{\theta\left(P_{+}-e\right) E\left(P_{+}, P_{2}\right)}\right)^{2} \frac{1}{4 \pi i} \oint_{C}\left(\frac{E\left(P, P_{2}\right) \theta(P-e)}{E\left(P, P_{+}\right) E\left(P, P_{-}\right)}\right)^{2}
$$

and

$$
N_{-1 / 2}^{(\lambda)}\left(P_{+}, P_{-}\right)=\frac{E\left(P_{+}, P_{-}\right)}{\theta\left(P_{+}-e\right) E\left(P_{+}, P_{2}\right)} .
$$

Let us now recall the definition of the contours $C_{\tau}$ [12]. On $\Sigma$ there exists a well-defined harmonic function of $Q: \operatorname{Re}\left(\int_{Q_{0}}^{Q} \omega^{g / 2}\right)\left(Q_{0} \in \Sigma\right.$ is an arbitrary reference point). The contours $C_{\tau}$ are defined as the level lines of this function, i.e.

$$
C_{\tau}=\left\{Q \in \Sigma: \operatorname{Re}\left(\int_{Q_{0}}^{Q} \omega^{g / 2}\right)=\tau\right\} .
$$

For $\tau \rightarrow \pm \infty$ the contours $C_{\tau}$ become small circles around $P_{\mp}$. The coordinate $\tau$ plays the role of (euclidean) time, and the level lines $C_{\tau}$ can be interpreted as the position of the string at a given time $\tau$. So we have a pictorial image of the string propagating on the Riemann surface, with as many splittings and joinings (interaction) as the genus $g$ of $\Sigma$. As for the orientation, $C_{\tau}$ as well as any integration contour separating $P_{+}$and $P_{-}$is understood henceforth to encircle $P_{+}$in an anticlockwise way.

The dual section of $f_{j}^{(\lambda)}, f_{(1-\lambda)}^{j}$, is defined by the following duality relation:

$$
\frac{1}{2 \pi i} \oint_{C_{\tau}} f_{i}^{(\lambda)} f_{(1-\lambda)}^{j}=\delta_{i}^{j}
$$

The bases defined before satisfy this relation with $f_{(1-\lambda)}^{j}=f_{-j}^{(1-\lambda)}$ [in the particular

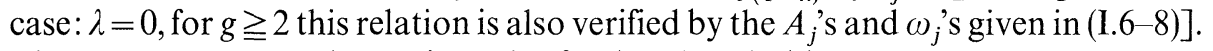
The constants were chosen in order for (I.12) to hold.

\section{Case of Half-Integer $\lambda$}

Let us now consider sections of $K^{\lambda}$ with a given spin structure $[\alpha, \beta]$. We are interested in two kinds of bases:

i) Basis for the space of tensors of weight $\lambda$ with the spin structure $[\alpha, \beta]$ which are holomorphic outside $P_{+}$and $P_{-}$and a slit from $P_{+}$to $P_{-}$("Ramond-type" bases);

ii) Basis for thespace of tensors of weight $\lambda$ with the spin structure $[\alpha, \beta]$ which are holomorphic outside $P_{+}$and $P_{-}$("Neveu-Schwarz(NS)-type" bases).

By Riemann-Roch theorem, there exists a unique (up to a normalization constant) section $f_{n}^{(\lambda)}$ which in neighborhoods of $P_{ \pm}$have the form (when the spin structure is odd, the following expression is slightly modified in the NS sector in the cases $\lambda=1 / 2$ with $|n|=1 / 2$ or $g=1$, see below)

$$
f_{n}^{(\lambda)}\left(z_{ \pm}\right)=a_{n}^{ \pm} z_{ \pm}^{ \pm n-s(\lambda)}\left(1+O\left(z_{ \pm}\right)\right)\left(d z_{ \pm}\right)^{\lambda}
$$


where $n$ takes integer values in the Ramond case i), and half-integer values in the NS case ii).

Even though (I.13) looks like (I.1), there is however a difference due to the fact that the indices $j$ or $n$, run in general over distinct values. Throughout this section indices $i, j, k$ will be used to label the elements of the bases for integer $\lambda$, and $l, m, n$ to label the elements of the bases for half-integer $\lambda$.

Let us now consider the NS sector with odd spin structure, and $\lambda=1 / 2$. If $n \neq \pm 1 / 2$, then Eq. (I.13) still holds. For $n= \pm 1 / 2$ we take the sections as follows:

$$
\begin{gathered}
f_{-1 / 2}^{(1 / 2)}\left(z_{ \pm}\right)=a_{-1 / 2}^{ \pm} z_{ \pm}^{-1}\left(1+O\left(z_{ \pm}\right)\right)\left(d z_{ \pm}\right)^{1 / 2}, \\
f_{1 / 2}^{(1 / 2)}\left(z_{ \pm}\right)=a_{1 / 2}^{ \pm}\left(1+O\left(z_{ \pm}\right)\right)\left(d z_{ \pm}\right)^{1 / 2} .
\end{gathered}
$$

Considering as before the NS sector, odd spin structure, but $g=1$, we can define for any half integer $\lambda$

$$
f_{n}^{(\lambda)}=\text { constant } A_{n} \eta^{\lambda}
$$

where we take the spin structure of $\eta^{1 / 2}$ odd.

The explicit construction of these bases is made in the same way as for integer $\lambda$, but now we have to take into account the spin structure. This is accomplished by introducing $\theta$-functions with characteristics $\left[\begin{array}{l}\alpha \\ \beta\end{array}\right]$. Then, we write

$$
f_{n}^{(\lambda)}(P)=N_{n}^{(\lambda)}\left(P_{+}, P_{-}\right) \frac{E\left(P, P_{+}\right)^{n-s(\lambda)}}{E\left(P, P_{-}\right)^{n+s(\lambda)}} \sigma(P)^{2 \lambda-1} \theta\left[\begin{array}{l}
\alpha \\
\beta
\end{array}\right](P+e(\lambda, n)),
$$

where

$$
\begin{gathered}
e(\lambda, n)=(n-s(\lambda)) P_{+}-(n+s(\lambda)) P_{-}+(1-2 \lambda) \Delta, \\
N_{n}^{(\lambda)}\left(P_{+}, P_{--}\right)=\frac{E\left(P_{+}, P_{-}\right)^{n+s(\lambda)} \sigma\left(P_{+}\right)^{2 n}}{\theta\left[\begin{array}{l}
\alpha \\
\beta
\end{array}\right]\left(P_{+}+e(\lambda, n)\right)} .
\end{gathered}
$$

For the particular case of $\left[\begin{array}{l}\alpha \\ \beta\end{array}\right]$ corresponding to an odd spin structure, NS sector and $\lambda=1 / 2$ we have for $|n|=1 / 2$,

$$
f_{1 / 2}^{(1 / 2)}(P)=N_{1 / 2}^{(1 / 2)}\left(P_{+}, P_{-}\right) \theta\left[\begin{array}{l}
\alpha \\
\beta
\end{array}\right]\left(P-P_{-}\right) / E\left(P, P_{-}\right),
$$

where

$$
N_{1 / 2}^{(1 / 2)}\left(P_{+}, P_{-}\right)=\frac{E\left(P_{+}, P_{-}\right) \sigma\left(P_{+}\right)}{\theta\left[\begin{array}{l}
\alpha \\
\beta
\end{array}\right]\left(P_{+}-P_{-}\right)}
$$

and

$$
f_{-1 / 2}^{(1 / 2)}(P)=N_{-1 / 2}^{(1 / 2)}\left(P_{ \pm}, R\right) \frac{E(P, R)}{E\left(P, P_{+}\right) E\left(P, P_{-}\right)} \theta\left[\begin{array}{l}
\alpha \\
\beta
\end{array}\right](P+e),
$$


where $e=R-P_{+}-P_{-}$, and

$$
N_{-1 / 2}^{(1 / 2)}\left(P_{ \pm}, R\right)=\frac{E\left(P_{+}, P_{-}\right)}{E\left(P_{+}, R\right) \sigma\left(P_{+}\right)} \theta\left[\begin{array}{l}
\alpha \\
\beta
\end{array}\right]\left(P_{+}+e\right),
$$

where $R$ is a generic fixed point. The dual bases are defined as in the $\lambda$ integer case. This completes the explicit construction of the $\mathrm{KN}$ bases.

The Structure Constants $C_{i j}^{s}$

As an immediate application, the expressions for the bases given above can be used to explicitly write the structure constants $C_{i j}^{s}$ of the Krichever-Novikov algebra [12]

$$
\left[e_{i}, e_{j}\right]=\sum_{s=-g_{0}}^{g_{0}} C_{i j}^{s} e_{i+j-s},
$$

where $e_{i} \equiv f_{i}^{(-1)}$ and $g_{0}=3 g / 2$. By integrating with the dual to $e_{i+j-s}$ we have

$$
C_{i j}^{s}=\frac{1}{2 \pi i} \oint_{C_{\tau}} \Omega^{i+j-s}\left[e_{i}, e_{j}\right],
$$

where $\Omega^{k} \equiv f_{(2)}^{k}$. Now we can insert the explicit formulas given by (I.6) for $\lambda=-1$ and its dual, and so obtain the $C_{i j}^{s}$ 's in terms of $\theta$-functions and their derivatives.

In [12] the $C_{i j}^{s}$, s were given only for $s=-g_{0}$ and $s=g_{0}(g>1)$ :

$$
C_{i j}^{g_{0}}=(j-i), \quad C_{i j}^{-g_{0}}=(i-j) \frac{\varphi_{i}^{(-1)-} \varphi_{j}^{(-1)-}}{\varphi_{i+j+g_{0}}^{(-1)-}} .
$$

For $s=-g_{0}+1, \ldots, g_{0}-1$, the structure constants will depend on the successive coefficients of the expansion of $e_{i}$ around $P_{+}$or $P_{-}$[cf.(I.1)], which can also be written in terms of $\theta$-functions and their derivatives using the formulas given above. For example

$$
\varphi_{i}^{(-1)^{-}}=(-1)^{i+g_{0}-1} E\left(P_{-}, P_{+}\right)^{2 i} \frac{\theta(P-+e(-1, i))}{\theta\left(P_{+}+e(-1, i)\right)}\left(\frac{\sigma\left(P_{-}\right)}{\sigma\left(P_{+}\right)}\right)^{2 \lambda-1} .
$$

\section{The Operator Formalism}

Any tensor $F^{(\lambda)}(P)\left(P \in C_{\tau}\right)$ of weight $\lambda$ which is smooth (or piecewise smooth) over $C_{\tau}$ can be expanded in the basis $\left\{f_{k}^{(\lambda)}\right\}[12,13]$,

$$
F^{(\lambda)}(P)=\sum_{k} a_{k} f_{k}^{(\lambda)}(P) .
$$

Therefore, if we have a tensor over $\Sigma$ which is smooth except possibly in $P_{ \pm}$, the expansion (II.1) will hold for any $C_{\tau},-\infty<\tau<\infty$, with coefficients generally depending on $\tau$. But if we consider a meromorphic $F^{(\lambda)}$ which is holomorphic outside $P_{ \pm}$, the coefficients $a_{k}$ will be $\tau$-independent. This is the case for $b-c$ systems, as the tensors $b$ and $c$ are supposed to satisfy $\bar{\partial} b(P)=0=\bar{\partial} c(P)$ everywhere except at $P_{ \pm}$(a discussion concerning the zero modes, i.e., the globally holomorphic tensors of the $b$ and $c$ type, will be given in Sect. IV). So let us expand 
them according to (II.1),

$$
\begin{gathered}
b(P)=\sum_{k} b_{k} f_{(\lambda)}^{k}(P), \\
c(P)=\sum_{k} c^{k} f_{k}^{(1-\lambda)}(P) .
\end{gathered}
$$

The summations run over the integers or half-integers depending on the case we are considering (cf. previous section).

Upon quantization, $b(P)$ and $c(P)$ become operators and satisfy canonical (anti-)commutation relations. Henceforth we will consider for simplicity the case of anticommuting $b$ and $c$ fields with integer $\lambda>1, g>1$. Then, the coefficients $b_{k}$ 's and $c^{k}$ 's will satisfy the standard anticommutation rules

$$
\begin{gathered}
\left\{b_{i}, c^{j}\right\}=\delta_{i}^{j}, \\
\left\{c^{i}, c^{j}\right\}=0=\left\{b_{i}, b_{j}\right\} .
\end{gathered}
$$

They are operators in a Fock space with vacuum state $\mid 0)_{\Sigma}$ satisfying the conditions

$$
\begin{aligned}
& \left.c^{k} \mid 0\right)_{\Sigma}=0, \quad k<s(1-\lambda), \\
& \left.b_{k} \mid 0\right)_{\Sigma}=0, \quad k \geqq s(1-\lambda) .
\end{aligned}
$$

We can represent the vacuum as the semi-infinite form

$$
\mid 0)_{\Sigma}=f_{(\lambda)}^{s(1-\lambda)} \wedge f_{(\lambda)}^{s(1-\lambda)+1} \wedge \ldots
$$

[from now on we drop the label $(\lambda)$ when it is not strictly necessary]. Then the action of $c^{k}$ and $b_{k}$ on the vacuum admits the explicit representation (right action)

$$
\begin{gathered}
b_{k}=f^{k} \wedge, \\
\left.\left.c^{k} \mid 0\right)_{\Sigma}=i_{f_{k}} \mid 0\right)_{\Sigma}=\sum_{j=s(1-\lambda)}^{\infty}(-1)^{j} i_{f_{k}}\left(f^{j}\right) f^{s(1-\lambda)} \wedge \ldots \wedge f^{j} \wedge \ldots,
\end{gathered}
$$

where $i_{f_{k}}$ denotes the usual antiderivation defined by

$$
i_{f_{k}}\left(f^{j}\right)=\frac{1}{2 \pi i} \underset{C_{\tau}}{f_{k}} f_{k} f^{j}=\delta_{h}^{j},
$$

and $^{`}$ denotes omission. Analogously we can define the dual vacuum ${ }_{\Sigma}(0 \mid$ by means of

$$
\begin{array}{ll}
{ }_{\Sigma}\left(0 \mid c^{k}=0,\right. & k \geqq s(1-\lambda), \\
{ }_{\Sigma}\left(0 \mid b_{k}=0,\right. & k<s(1-\lambda) .
\end{array}
$$

It can be usefully given the representation $[12,13]$

$$
{ }_{\Sigma}\left(0 \mid=f_{s(1-\lambda)} \wedge f_{s(1-\lambda)+1} \wedge \ldots,\right.
$$

$c^{k}$ and $b_{k}$ act on this semi-infinite form (left action) as

$$
c^{k}=f_{k} \wedge, \quad b_{k}=i_{f^{k}} .
$$

Moreover there exists a pairing such that

$$
{ }_{\Sigma}(0 \mid 0)_{\Sigma}=1 \text {. }
$$


It is now time to comment on the meaning of this vacuum and of the excitations which are created out of it or destroyed by the operators $c^{k}$ and $b_{k}$. First of all, we have to emphasize that both these operators and the vacuum state are globally defined over $\Sigma$, due to the fact that the $\mathrm{KN}$ bases are globally defined. In this sense our approach is different from the previous ones [3-7], where two vacua are generally used, one related to a disk singled out of the surface and the other related to the rest of $\Sigma$.

It is evident that the $c^{k}$ and $b_{k}$ modes are related in a complicated ( $g$-dependent) way to the usual $g=0$ modes, which are the string modes with associated particle interpretation. Let us find the relation between the genus $g$ modes and the genus zero modes. We pick a coordinate $z$ near $P_{+}, z\left(P_{+}\right)=0$, and the circle $C=\{|z|=1\}$. A basis for tensors of weight $\lambda$ over this circle is given by

$$
\begin{aligned}
f_{n}^{(1-\lambda)}(z) & =z^{n-1+\lambda}(d z)^{1-\lambda}, \\
f_{(\lambda)}^{n}(z) & =z^{-n-\lambda}(d z)^{\lambda},
\end{aligned}
$$

where $n$ is integer. The restrictions $\left\{\bar{f}_{j}^{(1-\lambda)}\right\}$ and $\left\{\bar{f}_{(\lambda)}^{j}\right\}$ of the bases $\left\{f_{j}^{(1-\lambda)}\right\}$ and $\left\{f_{(\lambda)}^{j}\right\}$ on $C$ are dense in the space of the corresponding tensors on $C$ [12]. Therefore, we can expand

where

$$
\begin{aligned}
\bar{f}_{j}^{(1-\lambda)} & =\sum_{n} A_{j}^{n}(1-\lambda) \tilde{f}_{n}^{(1-\lambda)}, \\
\tilde{f}_{n}^{(1-\lambda)} & =\sum_{j} B_{n}^{j}(1-\lambda) \bar{f}_{j}^{(1-\lambda)}, \\
\bar{f}_{(\lambda)}^{j} & =\sum_{n} C_{n}^{j}(\lambda) \tilde{f}_{(\lambda)}^{n}, \\
\tilde{f}_{(\lambda)}^{n} & =\sum_{j} D_{j}^{n}(\lambda) \bar{f}_{(\lambda)}^{j},
\end{aligned}
$$

$$
\begin{aligned}
& A_{j}^{n}(1-\lambda)=\frac{1}{2 \pi i} \oint_{C} \bar{f}_{j}^{(1-\lambda)} \tilde{f}_{(\lambda)}^{n}, \\
& B_{n}^{j}(1-\lambda)=\frac{1}{2 \pi i} \oint_{C} \bar{f}_{(\lambda)}^{j} \widetilde{f}_{n}^{(1-\lambda)}, \quad \text { etc. }
\end{aligned}
$$

It is easy to see that $A(1-\lambda)=B(1-\lambda)^{-1}, C(\lambda)=D(\lambda)^{-1}$, and $C(\lambda)=B(1-\lambda)$. The entries of the $A(1-\lambda)$ matrix vanish for $n<j+s(\lambda)-\lambda$, and are given by the coefficients of the Laurent tail in Eq. (I.1) otherwise. Similarly $B_{n}^{j}(1-\lambda)$ vanish if $n>j+s(\lambda)-\lambda$, and is otherwise given by the coefficients of the Laurent expansion of $\bar{f}_{(1-\lambda)}^{j}$ near $P_{+}$. We remark that in general the matrices $A(1-\lambda)$ and $B(1-\lambda)$ have an infinite number of non-vanishing entries and stress that they can be explicitly calculated.

Now we are able to calculate the relation between creation and annihilation operators on the sphere and on genus $g$ Riemann surfaces. Let $\bar{b}$ and $\bar{c}$ be the restrictions of $b$ and $c$ to the circle $C$, then we can consider both expansions

$$
\begin{aligned}
& \bar{b}(P)=\sum_{j} b_{j} \bar{f}_{(\lambda)}^{j}(P)=\sum_{n} \widetilde{b}_{n} \tilde{f}_{(\lambda)}^{n}(P), \\
& \bar{c}(P)=\sum_{j} c^{j} \bar{f}_{j}^{(1-\lambda)}(P)=\sum_{n} \tilde{c}^{n} \tilde{f}_{n}^{(1-\lambda)}(P) .
\end{aligned}
$$


It is easy to find the relation between the $\tilde{b}_{i}$ 's, $b_{i}$ 's, $\tilde{c}^{i}$ 's, and $c^{i}$ 's,

$$
\tilde{c}^{n}=\sum_{j} A_{j}^{n}(1-\lambda) c^{j}, \quad \tilde{b}_{n}=\sum_{j} B_{n}^{j}(1-\lambda) b_{j} .
$$

These are infinite combinations, however one can realize that when applied to $(0)_{\Sigma}$ only a finite combination survives due to (II.4) and the properties of the $A$ and $B$ matrices listed after Eq. (II.13). As a consequence this is true for any state constructed from $\mid 0)_{\Sigma}$ by applying a finite number of $\tilde{b}_{n}$ and $\tilde{c}^{n}$ operators. In particular we have

$$
\begin{array}{lll}
\left.\tilde{b}_{n} \mid 0\right)_{\Sigma}=0 & \text { for } & n+\lambda-1 \geqq 0, \\
\left.\tilde{c}^{n} \mid 0\right)_{\Sigma}=0 & \text { for } & n+\lambda-1<0 .
\end{array}
$$

Of course, we could have started from the $g=0$ vacuum $\mid 0)_{0}$ defined by Eq. (II.5) where $g=0$, and tried to find the action of $b_{k}$ and $c^{k}$ over it. We would have found Eq. (II.4) with $\mid 0)_{0}$ instead of $\left.\mid 0\right)_{\Sigma}$. The relation between $\left.\mid 0\right)_{0}$ and $\left.\mid 0\right)_{\Sigma}$ for a genus $g \neq 0$ $\Sigma$ can be reconstructed by means of the semi-infinite form representation of $(0)_{\Sigma}$ and the transformation of the basis elements. For example, we have for the quadratic differentials:

$$
\Omega^{j}=\widetilde{\Omega}^{j-g_{0}}+\sum_{n<j-g_{0}}^{\infty} B_{n}^{j}(2) \widetilde{\Omega}^{n} .
$$

In this connection see [3-7, 20].

It is now worth spending a few words about the connection between the vacuum defined here and a vacuum frequently used in the literature [3-7]. A definition of the vacuum $|0\rangle_{\Sigma}$ implicit in the path integral approach (see Sect. IV) is specified by means of (II.4) and

$$
\begin{array}{ll}
{ }_{\Sigma}<0 \mid c^{k}=0, & k>-s(1-\lambda), \\
{ }_{\Sigma}<0 \mid b_{k}=0, & k \leqq-s(1-\lambda),
\end{array}
$$

instead of (II.7). $|0\rangle_{\Sigma}$ and ${ }_{\Sigma}\langle 0|$ can be represented by two semi-infinite forms, given respectively by Eq. (II.5) and by

$$
{ }_{\Sigma}\langle 0|=f_{-s(1-\lambda)+1} \wedge f_{-s(1-\lambda)+2} \wedge \ldots,
$$

$c^{k}$ and $b_{k}$ acts as in Eq. (II.6) and Eq. (II.9). It is evident that

$$
{ }_{\Sigma}\langle 0 \mid 0\rangle_{\Sigma}=0
$$

and that ${ }_{\Sigma}\langle 0|$ and ${ }_{\Sigma}(0 \mid$ differ exactly by the dual bases elements of the $b$ zero modes for $\lambda>1$ and for $g \geqq 2$.

For $g=0, \lambda=2$, let us define

$$
|0\rangle_{0}=\widetilde{\Omega}^{-1} \wedge \widetilde{\Omega}^{0} \wedge \ldots, \quad{ }_{0}\langle 0|=\tilde{e}_{2} \wedge \tilde{e}_{3} \wedge \ldots,
$$

and assume

$$
|0\rangle_{0}^{+}={ }_{0}\langle 0|, \quad\left(c^{k}\right)^{+}=c^{-k}, \quad\left(b_{k}\right)^{+}=b_{-k} .
$$

These definitions exactly realize the prescription

$$
\left(c_{-1}|0\rangle_{0}\right)^{+}={ }_{0}\langle 0| c_{1} c_{0}
$$


introduced in [15] (remember that with respect to this reference $c_{k} \leftrightarrow c_{-k}$ ). It is therefore possible to interpret the pairing defined by the brackets ${ }_{\Sigma}\langle\mid\rangle_{\Sigma}$ as a scalar product.

\section{Propagators for $b-c$ Systems}

Propagators for Integer $\lambda$

Let us first consider the $g>1, \lambda>1$ case. The propagator $S(P, Q)$ is defined as

$$
S(P, Q)={ }_{\Sigma}(0|T\{b(P) c(Q)\}| 0)_{\Sigma}= \begin{cases}\Sigma_{\Sigma}(0|b(P) c(Q)| 0)_{\Sigma}, & \tau_{P}>\tau_{Q} \\ -{ }_{\Sigma}(0|c(Q) b(P)| 0)_{\Sigma}, & \tau_{Q}>\tau_{P},\end{cases}
$$

where $\tau_{P}\left(\tau_{Q}\right)$ means the value of $\tau$ at the point $P(Q)$. By inserting (II.2) into (III.1), and using (II.3, 4, 7) we easily obtain

$$
S(P, Q)= \begin{cases}\sum_{k=s(1-\lambda)}^{\infty} f_{(\lambda)}^{k}(P) f_{k}^{(1-\lambda)}(Q), & \tau_{P}>\tau_{Q} \\ -\sum_{k=-\infty} f_{(\lambda)}^{k}(P) f_{k}^{(1-\lambda)}(Q), & \tau_{Q}>\tau_{P} .\end{cases}
$$

Now we would like to give a more compact expression for this Szegö kernel. Eventually we will find that $S(P, Q)$ reduces to the known result (see $[4,21,22]$ )

$$
S(P, Q)=\frac{1}{E(P, Q)}\left(\frac{E\left(P, P_{-}\right)}{E\left(Q, P_{-}\right)}\right)^{(2 \lambda-1)(g-1)}\left(\frac{\sigma(P)}{\sigma(Q)}\right)^{(2 \lambda-1)} \frac{\theta(Q-P+u(\lambda))}{\theta(u(\lambda))},
$$

where

$$
u(\lambda)=-(2 \lambda-1)(g-1) P_{-}+(2 \lambda-1) \Delta .
$$

A check that Eqs. (III.3) and (III.2) coincide is the following. Consider the propagator to be a tensor $F^{(1-\lambda)}(Q)$ of weight $1-\lambda$ depending on $Q$, at fixed $P$. Then it can be expanded in terms of the basis $\left\{f_{k}^{(1-\lambda)}\right\}$,

$$
F^{(1-\lambda)}(Q)=\sum_{k} a_{k} f_{k}^{(1-\lambda)}(Q)
$$

Multiplying by $f_{(\lambda)}^{k}$ and integrating over $C_{\tau}$ we obtain

$$
a_{k}=\frac{1}{2 \pi i} \oint_{C_{\tau}} F^{(1-\lambda)} f_{(\lambda)}^{k} .
$$

Now we can use Eq. (III.3) and the explicit expressions for $f_{(\lambda)}^{k}$ in order to arrive at Eq. (III.2). In fact, solving the integral (III.5) we obtain

$$
\begin{aligned}
& a_{k}=\left\{\begin{array}{ll}
f_{(\lambda)}^{k}, & k \geqq s(1-\lambda) \\
0, & k<s(1-\lambda)
\end{array}, \quad \tau_{Q}<\tau_{P},\right. \\
& a_{k}=\left\{\begin{array}{ll}
0 & k \geqq s(1-\lambda) \\
-f_{(\lambda)}^{k}, & k<s(1-\lambda)
\end{array}, \quad \tau_{P}<\tau_{Q},\right.
\end{aligned}
$$

in agreement with Eq. (III.2). 
It is instructive to see how one can use a heuristic argument to pass from (III.2) to the compact form (III.3). This consists in looking at the behaviour of the sums (III.2), and then identifying the zeroes and poles of $S(P, Q)$. After that, one uses Riemann-Roch theorem to prove the existence and uniqueness of sections with such behaviours, and the explicit expression (III.3) easily follows.

Let us first consider the point $P$ fixed. From (III.2) we get

$$
F^{(1-\lambda)}(Q)= \begin{cases}\sum_{k=s(1-\lambda)}^{\infty} a_{k} f_{k}^{(1-\lambda)}(Q), & \tau_{P}>\tau_{Q} \\ s(1-\lambda)-1 & \\ -\sum_{k=-\infty} a_{k} f_{k}^{(1-\lambda)}(Q), & \tau_{Q}>\tau_{P} .\end{cases}
$$

In order to see the behaviour of $F^{(1-\lambda)}(Q)$ on the Riemann surface, we use Eq. (I.1). It follows that

$$
\begin{aligned}
& Q \rightarrow P_{+} \Rightarrow F^{(1-\lambda)}(Q) \text { is holomorphic non-zero; } \\
& Q \rightarrow P_{-} \Rightarrow F^{(1-\lambda)}(Q) \text { has a pole of order }(2 \lambda-1)(g-1) ; \\
& Q \rightarrow P \quad \Rightarrow F^{(1-\lambda)}(Q) \text { has a simple pole. }
\end{aligned}
$$

Proceeding likewise, but at fixed $Q$, the propagator can be considered as a tensor of weight $\lambda$ which we denote by $F_{(\lambda)}^{+}$

$$
F_{(\lambda)}^{+}(P)=\left\{\begin{array}{l}
\sum_{k=s(1-\lambda)}^{\infty} b_{k} f_{(\lambda)}^{k}(P), \quad \tau_{P}>\tau_{Q} \\
s(1-\lambda)-1 \\
-\sum_{k=-\infty} b_{k} f_{(\lambda)}^{k}(P), \quad \tau_{Q}>\tau_{P} .
\end{array}\right.
$$

Similarly, from Eq. (III.4) we extract the behaviour of $F_{(\lambda)}^{+}(P)$,

$P \rightarrow P_{+} \Rightarrow F_{(\lambda)}^{+}(P)$ is holomorphic non-zero;

$P \rightarrow P_{-} \Rightarrow F_{(\lambda)}^{+}(P)$ is holomorphic with a zero of order $(2 \lambda-1)(g-1) ; \quad$ (III.10)

$P \rightarrow Q_{-} \Rightarrow F_{(\lambda)}^{+}(P)$ has a simple pole.

Riemann-Roch theorem tells us that $F^{(1-\lambda)}(Q)$ and $F_{(\lambda)}^{+}(P)$ are uniquely determined up to a constant $C_{1}\left(P, P_{ \pm}\right)$and $C_{2}\left(Q, P_{ \pm}\right)$respectively. So, there remains an indetermination by a constant $C\left(P_{ \pm}\right)$. The propagator $S(P, Q)$ is now easily found from (III.8) and (III.10), and from the requirement of correct dimensionality and single-valuedness,

$$
S(P, Q)=\frac{C\left(P_{ \pm}\right)}{E(P, Q)}\left(\frac{E\left(P, P_{-}\right)}{E\left(Q, P_{-}\right)}\right)^{(2 \lambda-1)(g-1)}\left(\frac{\sigma(P)}{\sigma(Q)}\right)^{(2 \lambda-1)} \frac{\theta(Q-P+u(\lambda))}{\theta(u(\lambda))},
$$

where

$$
u(\lambda)=-(2 \lambda-1)(g-1) P_{-}+(2 \lambda-1) \Delta .
$$

The constant $C\left(P_{ \pm}\right)$can be determined by making $P \rightarrow P_{-}$and $Q \rightarrow P_{+}$, where the propagator has a zero of order $(2 \lambda-1)(g-1)$,

$$
S(P, Q)=\sum_{k=s(1-\lambda)}^{\infty} f_{(\lambda)}^{k}(P) f_{k}^{(1-\lambda)}(Q) \underset{Q \rightarrow P_{+}}{\stackrel{P \rightarrow P}{\approx}} f_{(\lambda)}^{s(1-\lambda)}(P) f_{s(1-\lambda)}^{(1-\lambda)}(Q) .
$$


Inserting the explicit expressions for $f_{(\lambda)}^{k}$ and $f_{k}^{(1-\lambda)}$ we obtain

$$
S(P, Q) \underset{Q \rightarrow P_{+}}{\stackrel{P \rightarrow P-}{\approx}} \frac{E\left(P, P_{-}\right)^{(2 \lambda-1)(g-1)}}{E\left(P_{+}, P_{-}\right)^{(2 \lambda-1)(g-1)+1}}\left(\frac{\sigma\left(P_{-}\right)}{\sigma\left(P_{+}\right)}\right)^{(2 \lambda-1)} \frac{\theta\left(P_{-}-P_{+}+u\right)}{\theta(u)} .
$$

Now, comparing (III.13) with (III.11) we conclude that $C\left(P_{ \pm}\right)=1$.

Finally a remark. Had we chosen the vacuum state to be the state which is annihilated by the negative frequency modes of $c(P)$ and $b(P)$ with $P$ near to $P_{-}$, then we would have found a similar result for $S(P, Q)$ with $P_{+}$instead of $P_{-}$. A useful relation to prove this statement is

$$
f_{k}^{(\lambda)}\left(P ; P_{+}, P_{-}\right)=c f_{-k}^{(\lambda)}\left(P ; P_{-}, P_{+}\right)
$$

where $c$ is a constant independent of $P$.

Let us come now to the $\lambda=1$ case. We have already seen that the bases $\left\{A_{i}\right\},\left\{\omega_{i}\right\}$ are slightly modified with respect to the generic $f_{k}^{(\lambda)}$, Eq. (I.6). The vacuum state in this case is defined by the conditions

$$
\begin{array}{ll}
\left.c^{k} \mid 0\right)_{\Sigma}=0, & \mathrm{k} \leqq \mathrm{g} / 2, \\
b_{k}(0)_{\Sigma}=0, & k>g / 2 .
\end{array}
$$

So, the propagator is

$$
S(P, Q)= \begin{cases}\sum_{k=g / 2+1}^{\infty} \omega^{k}(P) A_{k}(Q), & \tau_{P}>\tau_{Q} \\ -\sum_{k=-\infty}^{g / 2} \omega^{k}(P) A_{k}(Q), & \tau_{Q}>\tau_{P} .\end{cases}
$$

The summations in (III.15) can be performed by the two methods explained before. We will not repeat the computation, since it follows the same lines as above. We just quote here the result which agrees with the well-known Szegö kernel for $\lambda=1$ [21]

$$
S(P, Q)=\frac{E\left(Q, P_{+}\right) \theta(Q-P-u) \theta\left(P-P_{+}-u\right)}{E(P, Q) E\left(P, P_{+}\right) \theta(u) \theta\left(Q-P_{+}-u\right)},
$$

where $u=g P_{-}-P_{+}-\Delta$.

Finally, let us consider the genus one case. The vacuum state is defined by the conditions

$$
\begin{array}{ll}
\left.c^{k} \mid 0\right)_{\Sigma}=0, & k \leqq 1 / 2, \\
b_{k}(0)_{\Sigma}=0, & k>1 / 2 .
\end{array}
$$

The propagator can be computed in much the same way as for the previous cases, and we obtain

$$
S(P, Q)=\frac{1}{E(P, Q)} \frac{E\left(P, P_{-}\right) E\left(Q, P_{+}\right)}{E\left(P, P_{+}\right) E\left(Q, P_{-}\right)}\left(\frac{\sigma(P)}{\sigma(Q)}\right)^{(2 \lambda-1)} \frac{\theta(Q-P+u)}{\theta(u)},
$$

where $u=P_{+}-P_{-}+(2 \lambda-1) \Delta$. 


\section{Propagators for Half-Integer $\lambda$}

These kind of propagators are of interest in the study of conformal field theories on Riemann surfaces, and in particular, in superstring theory, where we have matter with $\lambda=1 / 2$ and reparametrization ghosts with $\lambda=2$ as a anticommuting system, as well as superconformal ghost $(\beta, \gamma)$ as a commuting system with $\lambda=3 / 2$.

All of the results we have presented are easily extended to the commuting case and it is immediate to show that the propagator

$$
\begin{aligned}
S_{\beta \gamma}(P, Q) & ={ }_{\Sigma}(0|T\{\beta(P) \gamma(Q)\}| 0)_{\Sigma} \\
& ={ }_{\Sigma}(0|\beta(P) \gamma(Q)| 0)_{\Sigma} \theta\left(\tau_{P}-\tau_{Q}\right)+{ }_{\Sigma}(0|\gamma(Q) \beta(P)| 0)_{\Sigma} \theta\left(\tau_{Q}-\tau_{P}\right)
\end{aligned}
$$

gives the same $S(P, Q)$ as in the fermion case.

For half-integer $\lambda$ the only subtleties which arise in the computation of $S(P, Q)$ come from the presence of branch points in the Ramond sector, though these branch points are absent in integrals of the type (III.5) [this is due to the fact that in the Ramond sector both $F^{(1-\lambda)}$ and $f_{(\lambda)}^{j}$ in Eq. (III.5) have branch points in $\left.P_{ \pm}\right]$. So, we limit ourselves to give the results:

$g \geqq 2$, NS sector, $\lambda \neq 1 / 2$,

$$
S(P, Q)=\frac{1}{E(P, Q)}\left(\frac{E\left(P, P_{-}\right)}{E\left(Q, P_{-}\right)}\right)^{(2 \lambda-1)(g-1)}\left(\frac{\sigma(P)}{\sigma(Q)}\right)^{(2 \lambda-1)} \frac{\theta\left[\begin{array}{l}
\alpha \\
\beta
\end{array}\right](Q-P+u(\lambda))}{\theta\left[\begin{array}{l}
\alpha \\
\beta
\end{array}\right](u(\lambda))},
$$

where

$$
u(\lambda)=-(2 \lambda-1)(g-1) P_{-}+(2 \lambda-1) \Delta,
$$

$g \geqq 2$, Ramond sector,

$$
\begin{aligned}
S(P, Q)= & \frac{1}{E(P, Q)}\left(\frac{E\left(P, P_{-}\right)}{E\left(Q, P_{-}\right)}\right)^{(2 \lambda-1)(g-1)+1 / 2}\left(\frac{\sigma(P)}{\sigma(Q)}\right)^{(2 \lambda-1)} \\
& \times\left(\frac{E\left(Q, P_{+}\right)}{E\left(P, P_{+}\right)}\right)^{1 / 2} \theta \frac{\left[\begin{array}{l}
\alpha \\
\beta
\end{array}\right](Q-P+u(\lambda))}{\theta\left[\begin{array}{l}
\alpha \\
\beta
\end{array}\right](u(\lambda))}
\end{aligned}
$$

where

$$
u(\lambda)=1 / 2\left(P_{+}-P_{-}\right)-(2 \lambda-1)(g-1) P_{-}+(2 \lambda-1) \Delta .
$$

In the $\lambda=1 / 2$ case, formulas (III.20,21) still hold, except when the spin structure is odd and the sector is NS, for which we have

$$
S(P, Q)=\frac{E\left(P, P_{-}\right) E\left(Q, P_{+}\right) \theta\left[\begin{array}{l}
\alpha \\
\beta
\end{array}\right]\left(Q-P+P_{+}-P_{-}\right)}{E(P, Q) E\left(P, P_{+}\right) E\left(Q, P_{-}\right) \theta\left[\begin{array}{l}
\alpha \\
\beta
\end{array}\right]\left(P_{+}-P_{-}\right)} .
$$


If $g=1$, and the spin structure is odd, the propagator in the NS sector for any half-integer $\lambda$ is given by

$$
S(P, Q)=\frac{1}{E(P, Q)} \frac{E\left(P, P_{-}\right)}{E\left(P, P_{+}\right)} \frac{E\left(Q, P_{+}\right)}{E\left(Q, P_{-}\right)}\left(\frac{\sigma(P)}{\sigma(Q)}\right)^{(2 \lambda-1)} \frac{\theta\left[\begin{array}{l}
\alpha \\
\beta
\end{array}\right](Q-P+u(\lambda))}{\theta\left[\begin{array}{l}
\alpha \\
\beta
\end{array}\right](u(\lambda))},
$$

where

$$
u(\lambda)=P_{+}-P_{-}+(2 \lambda-1) \Delta
$$

For any other case with $g=1$, the propagator is given by Eqs. (III.20, 21).

We finally make some remarks concerning the $N$-point correlation functions. They can be calculated by using Wick's theorem. The only non-vanishing correlation functions are of the form ${ }_{\Sigma}\left(0\left|T\left\{\prod_{i=1}^{N}\left(b\left(P_{i}\right) c\left(Q_{i}\right)\right)\right\}\right| 0\right)_{\Sigma}$. The rule to
calculate them is

$$
\Sigma_{\Sigma}\left(0\left|T\left\{\prod_{i=1}^{N}\left(b\left(P_{i}\right) c\left(Q_{i}\right)\right)\right\}\right| 0\right)_{\Sigma}=\left\{\begin{array}{l}
\sum_{\sigma}(-)^{\sigma} \prod_{i=1}^{N}\left({ }_{\Sigma}\left(0\left|T\left\{b\left(P_{i}\right) c\left(Q_{i}\right)\right\}\right| 0\right)_{\Sigma}\right) \\
\sum_{\sigma} \prod_{i=1}^{N}\left(_{\Sigma}\left(0\left|T\left\{b\left(P_{i}\right) c\left(Q_{i}\right)\right\}\right| 0\right)_{\Sigma}\right)
\end{array}\right.
$$

corresponding to an anticommuting (up) or commuting (down) $b-c$ system; $\sigma$ runs over all permutations.

\section{Some Remarks Concerning Zero Modes}

\section{Zero Modes and Teichmüller Deformations}

In this section we discuss various questions connected with zero modes of the $\bar{\partial}$-operators.

It is interesting to note that the $\mathrm{KN}$ bases have among their elements the zero modes for $\lambda$-differentials, which are by definition the holomorphic sections of $K^{\lambda}$.

For example, we observe that from the explicit expressions given in Sect. I, the basis of meromorphic vector fields has three modes for $i= \pm 1,0$ when $g=0$, one zero mode when $g=1$ (corresponding to $i=1 / 2$ ), and no zero mode if $g \geqq 2$.

It is a well-known result that the number of zero modes of two-differentials, sometimes called quadratic differentials, coincides with the dimension of the moduli space. In fact, for $\lambda=2$ Eq. (I.1) becomes

$$
\Omega_{j}\left(z_{ \pm}\right)=\varphi_{j}^{(2) \pm} z_{ \pm}^{ \pm j-2+g_{0}}\left(1+O\left(z_{ \pm}\right)\right)\left(d z_{ \pm}\right)^{2} .
$$

This is a zero mode provided that $|j| \leqq g_{0}-2$; therefore there are $3 g-3$ quadratic differentials for $g \geqq 2$ and no zero modes for $g=0$.

If $g=1$ there is only one holomorphic section of $K^{\lambda}$ for any $\lambda \in Z$ [that labeled by $i=1 / 2$ in Eq. (I.10)].

On the other hand, we know that the number of zero modes of the $3 / 2$ differentials plus the number of the quadratic differentials gives the dimension of 
the supermoduli space. In fact, for $\lambda=3 / 2$ we obtain [cf. Eq. (I.13)]

$$
f_{n}^{(3 / 2)}\left(z_{ \pm}\right)=a_{n}^{(3 / 2) \pm} z_{ \pm}^{ \pm n+g-3 / 2}\left(1+O\left(z_{ \pm}\right)\right)\left(d z_{ \pm}\right)^{3 / 2},
$$

from which we observe the existence of $2 g-2$ zero modes.

The explicit global expressions of the zero modes can be obtained from the formulas of Sect. I. As an example, let us write the basis of quadratic differentials $(\lambda=2)$,

$$
\Omega_{j}(P)=N_{j}^{(2)}\left(P_{+}, P_{-}\right) \frac{E\left(P, P_{+}\right)^{j+g_{0}-2}}{E\left(P, P_{-}\right)^{j-g_{0}+2}} \sigma(P)^{3} \theta(P+e(2, j))
$$

where

$$
e(2, j)=\left(j+g_{0}-2\right) P_{+}-\left(j-g_{0}+2\right) P_{-}-34
$$

and

$$
N_{j}^{(2)}\left(P_{+}, P_{-}\right)=\frac{E\left(P_{+}, P_{-}\right)^{j-g_{0}+2} \sigma\left(P_{+}\right)^{-3} h\left(P_{+}\right)^{3 g}}{\theta\left(\left(j+g_{0}-1\right) P_{+}-\left(j-g_{0}+2\right) P_{-}-3 \lambda \Delta\right)}
$$

for $j=-g_{0}+2, \ldots, g_{0}-2, g_{0}=3 g / 2$.

The dual to the $\Omega_{j}$ 's form a basis for the Beltrami differentials $\mu^{i}$. They obey the duality relation

$$
\frac{1}{2 \pi i} \int_{\Sigma} \mu_{i} \Omega^{j}=\delta_{i}^{j}
$$

In order to discuss the deformations of the complex structure of $\Sigma$ we will closely follow the second reference in [4] (see also [22, 23]). The vector fields $e_{i}$ with $|i| \leqq g_{0}-2$ can be used to generate Teichmüller deformations of the Riemann surface in the following way. Divide the Riemann surface in two parts $\Sigma^{+}$and $\Sigma^{-}$ containing $P_{+}$and $P_{-}$respectively such that $\Sigma^{+}$be a small disk whose center is $P_{+}$ and $\Sigma^{+} \cap \Sigma^{-}=A$, where $A$ is an annulus. Take a local coordinate $z$ on the disk. We can use the vector field $e_{i}$ to obtain a new Riemann surface as follows. We deform $A \rightarrow A^{\prime}$ by

$$
z \rightarrow z+\varepsilon \tilde{e}_{i}, \quad z \in A, \quad \varepsilon \in C,
$$

where $e_{i}=\tilde{e}_{i}(z) \partial_{z}$. Now $\Sigma^{-}$is glued to the disk $\Sigma^{+}$by identifying the new annulus with the previous collar on $\Sigma^{+}$. This new Riemann surface is not analytically equivalent to the old one when $e_{i}$ has poles both in $P_{+}$and $P_{-}$which corresponds to $|i| \leqq g_{0}-2$.

Under the infinitesimal deformation (IV.5) the metric transforms to

$$
\gamma(\mu) \propto\left|d z+\varepsilon \mu_{i} d \bar{z}\right|^{2},
$$

where

$$
\mu_{i}(P)=\left\{\begin{array}{lll}
\bar{\partial} \tilde{e}_{i} & \text { if } & P \in \Sigma^{+} \\
0 & \text { if } & P \in \Sigma^{-}-A
\end{array}\right.
$$

Integrating by parts we observe that the Beltrami differentials defined by (IV.7) satisfy (IV.4). 
Now we are able to give the explicit expression for the variation of the period matrix $\Omega$ under Teichmüller deformations. Under the deformation of the complex structure given by (IV.7) we have [22]

$$
\delta_{k} \Omega_{i j} \equiv-\int_{\Sigma} \eta_{i} \eta_{j} \mu_{k}=-\oint \eta_{i} \eta_{j} e_{k},
$$

where the integration contour separates $P_{+}$and $P_{-}$. Then it is easy to see that the variation $\delta_{k} \Omega_{i j}$ vanishes if $|k| \geqq g_{0}-1$. Now suppose $v$ is a linear combination of meromorphic vector fields $e_{k}$ of the $\mathrm{KN}$ basis. Then the most general infinitesimal variation of the period matrix is given by Eq. (IV.8) with $e_{k}$ replaced by (for $g>1$ )

$$
v=\sum_{k=-g_{0}+2}^{g_{0}-2} y_{k} e_{k}
$$

where

$$
\begin{aligned}
e_{k}(P) & =N_{k}^{(-1)}\left(P_{+}, P_{-}\right) \frac{E\left(P, P_{+}\right)^{k-g_{0}+1}}{E\left(P, P_{-}\right)^{k+g_{0}-1}} \sigma(P)^{-3} \theta(P+u(k)), \\
u(k) & =\left(k-g_{0}+1\right) P_{+}-\left(k+g_{0}-1\right) P_{-}+3 \Delta .
\end{aligned}
$$

The normalization constant can be chosen as in (I.6). For the $g=1$ case, see Sect. I.

\section{Connection with the Path Integral Approach}

Zero modes enter in an essential way also in a definition of the vacuum state which is mutated from the path integral approach. It would be natural to ask that $b(P)|0\rangle_{\Sigma}, c(P)|0\rangle_{\Sigma}$ be finite in $P_{+}$, and ${ }_{\Sigma}\langle 0| b(P),{ }_{\Sigma}\langle 0| c(P)$ be finite in $P_{-}$. This leads for $\lambda>1$ and $g>1$ to

$$
\begin{array}{ll}
c^{k}|0\rangle_{\Sigma}=0, & k<s(1-\lambda), \\
b_{k}|0\rangle_{\Sigma}=0, & k \geqq s(1-\lambda),
\end{array}
$$

and

$$
\begin{array}{ll}
{ }_{\Sigma}<0 \mid c^{k}=0, & k>-s(1-\lambda), \\
{ }_{\Sigma}<0 \mid b_{k}=0, & k \leqq-s(1-\lambda),
\end{array}
$$

as in Sect. 2. If we define the propagator by

$$
\langle b(P) c(Q)\rangle={ }_{\Sigma}\langle 0|T\{b(P) c(Q)\}| 0\rangle_{\Sigma},
$$

then we would obtain a vanishing result. This is because the vacuum defined above gives in general ${ }_{\Sigma}\langle 0 \mid 0\rangle_{\Sigma}=0$, as can be seen by using the algebra. For example, for even $g$ and anticommuting $c$ and $b$ we have

$$
0={ }_{\Sigma}\left\langle 0\left|b_{0} c^{0}\right| 0\right\rangle_{\Sigma}={ }_{\Sigma}\langle 0 \mid 0\rangle_{\Sigma}-{ }_{\Sigma}\left\langle 0\left|c^{0} b_{0}\right| 0\right\rangle_{\Sigma}={ }_{\Sigma}\langle 0 \mid 0\rangle_{\Sigma} .
$$

This fact is similar to what takes place in the path integral approach,

$$
Z=\int[d b d c] \exp (-S[b, c])=0,
$$

because of the presence of the zero modes. 
In order to obtain meaningful results the partition function $Z$ is redefined by inserting as many $b$ and $c$ fields as the number $N$ and $M$ of the corresponding zero modes, that is (for any $\lambda$ and $g$ )

$$
Z\left(z_{1}, \ldots, z_{N} ; w_{1}, \ldots, w_{M}\right)=\int[d b d c] b\left(z_{1}\right) \ldots b\left(z_{N}\right) c\left(w_{1}\right) \ldots c\left(w_{M}\right) \exp (-S[b, c]) .
$$

A correlation function is then defined with respect to this measure

$$
\begin{aligned}
& \left\langle b\left(P_{1}\right) \ldots b\left(P_{r}\right) c\left(Q_{1}\right) \ldots c\left(Q_{s}\right)\right\rangle=Z\left(z_{1}, \ldots, z_{N} ; w_{1}, \ldots, w_{M}\right)^{-1} \\
& \quad \times \int[d b d c] b\left(z_{1}\right) \ldots c\left(w_{1}\right) \ldots b\left(P_{1}\right) \ldots c\left(Q_{1}\right) \ldots \exp (-S[b, c]) .
\end{aligned}
$$

In our operator formalism we proceed likewise and define a correlation function in the following way (throughout the rest of this section we drop the label $\Sigma$ from the vacuum):

$$
\left\langle b\left(P_{1}\right) \ldots b\left(P_{r}\right) c\left(Q_{1}\right) \ldots c\left(Q_{s}\right)\right\rangle=\frac{\left\langle 0\left|T\left\{b\left(z_{1}\right) \ldots c\left(w_{1}\right) \ldots b\left(P_{1}\right) \ldots c\left(Q_{1}\right) \ldots\right\}\right| 0\right\rangle}{\left\langle 0\left|T\left\{b\left(z_{1}\right) \ldots c\left(w_{1}\right) \ldots\right\}\right| 0\right\rangle}
$$

We will show that $(I V .12,13)$ lead to the same results and also are in agreement with those of Sect. III, provided we identify at the end of the computations the points $z_{i}$ with $P_{-}$and $w_{i}$ with $P_{+}$.

Let us first consider the case $\lambda>1$ for $g \geqq 2$. The propagator is then defined according to (IV.13),

$$
\langle b(P) c(Q)\rangle=\frac{\left\langle 0\left|T\left\{b\left(z_{1}\right) \ldots b\left(z_{N}\right) b(P) c(Q)\right\}\right| 0\right\rangle}{\left\langle 0\left|T\left\{b\left(z_{1}\right) \ldots b\left(z_{N}\right)\right\}\right| 0\right\rangle},
$$

where $N=-2 s(\lambda)+1$.

Let us choose $z_{1}, \ldots, z_{N}$ such that $\tau_{1}>\ldots>\tau_{N}$; then we have

$$
\begin{aligned}
& \left\langle 0\left|T\left\{b\left(z_{1}\right) \ldots b\left(z_{N}\right)\right\}\right| 0\right\rangle=\left\langle 0\left|b\left(z_{1}\right) \ldots b\left(z_{N}\right)\right| 0\right\rangle \\
& \quad=\operatorname{det}\left\|f^{i}\left(z_{j}\right)\right\|\left\langle 0\left|b_{+s(\lambda)} \ldots b_{-s(\lambda)}\right| 0\right\rangle, \quad i=s(\lambda), \ldots,-s(\lambda) .
\end{aligned}
$$

Let us take now $z_{i}$ near $P_{-}$and consider first $\tau_{P}>\tau_{Q}$ so that

$$
\begin{aligned}
& \left\langle 0\left|T\left\{b\left(z_{1}\right) \ldots b\left(z_{N}\right) b(P) c(Q)\right\}\right| 0\right\rangle=\left\langle 0\left|b\left(z_{1}\right) \ldots b\left(z_{N}\right) b(P) c(Q)\right| 0\right\rangle \\
& \quad=\sum_{\left\{i_{k}\right\}, j, k}\left\langle 0\left|b_{i_{1}} \ldots b_{i_{N}} b_{j} c^{k}\right| 0\right\rangle f^{i_{1}}\left(z_{1}\right) \ldots f^{i_{N}}\left(z_{N}\right) f^{j}(P) f_{k}(Q) \\
& \quad=\sum_{\text {perm. }}(-)^{P}\left\langle 0\left|b\left(z_{1}\right) \ldots \check{b}\left(z_{i}\right) \ldots b\left(z_{N}\right) b(P)\right| 0\right\rangle \sum_{j=s(1-\lambda)}^{\infty} f^{j}\left(z_{i}\right) f_{j}(Q),
\end{aligned}
$$

where $z_{i}$ takes the values $z_{1}, \ldots, z_{N}, P$. In a similar way one calculates the contribution for $\tau_{P}<\tau_{Q}$. The final result is given by

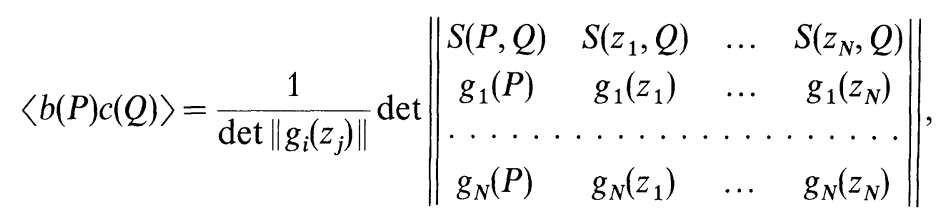

where $g_{1}, \ldots, g_{N}$ stands for $f^{+s(\lambda)}, \ldots, f^{-s(\lambda)}$. 
By taking the limit $z_{i} \rightarrow P_{-}$, for which $S\left(z_{i}, Q\right)=0$ we get

$$
\lim _{z_{\imath} \rightarrow P_{-}}\langle b(P) c(Q)\rangle=S(P, Q),
$$

as it was asserted.

Let us consider as another example the case $\lambda=1$. We obtain

$$
\begin{aligned}
& \langle b(P) c(Q)\rangle=\frac{1}{\operatorname{det}\left\|\omega_{i}\left(z_{j}\right)\right\|}
\end{aligned}
$$

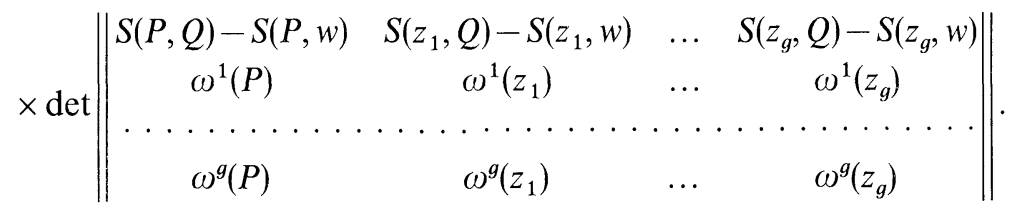

In the limit $z_{i} \rightarrow P_{-}, w \rightarrow P_{+}$, we recover the expected result

$$
\lim _{z_{\imath} \rightarrow P_{-}, w \rightarrow P_{+}}\langle b(P) c(Q)\rangle=S(P, Q) .
$$

\section{Appendix A}

Here we summarize the notation about theta functions [24]. Given the $g$ holomorphic differentials $\eta_{i}$ with the standard normalization around the basis of homology of the Riemann surface $\Sigma$,

$$
\oint_{a_{j}} \eta_{i}=\delta_{i j}, \quad \oint_{b_{j}} \eta_{i}=\Omega_{i j}, \quad \operatorname{Im}(\Omega)>0,
$$

the $\theta$-function with characteristics $\left[\begin{array}{l}\alpha \\ \beta\end{array}\right]$ associated to $\Sigma$ is

$$
\begin{aligned}
\theta\left[\begin{array}{l}
\alpha \\
\beta
\end{array}\right](z) & =\sum_{N \in Z^{g}} \exp \left(i \pi(N+\alpha)^{t} \Omega(N+\alpha)+i 2 \pi(N+\alpha)^{t}(z+\beta)\right) \\
& =\exp \left(i \pi \alpha^{t} \Omega \alpha+i 2 \pi \alpha^{t}(z+\beta)\right) \theta(z+\beta+\Omega \alpha), \\
\theta(z) & =\theta\left[\begin{array}{l}
0 \\
0
\end{array}\right](z), \quad z \in C^{g}, \quad \alpha, \beta, \in R^{g} .
\end{aligned}
$$

The Jacobian torus is defined by $J(\Sigma)=C^{g} / \Gamma(\Sigma)$ where the period lattice $\Gamma(\Sigma)$ is

$$
\Gamma(\Sigma)=\left\{v \in C^{g}: v=n+\Omega m,(n, m) \in Z^{2 g}\right\} .
$$

The set $\Theta=\{z: \theta(z)=0\}$ is a variety of complex codimension one in $J(\Sigma)$ called $\Theta$-divisor.

When the characteristics $\alpha_{i}, \beta_{i}$ are 0 or $1 / 2$, the corresponding $\theta$-function (A.2), known as first order theta function, is even or odd depending on the parity of $4 \alpha^{t} \beta$. The theta function is the unique holomorphic section of a holomorphic line bundle on $J(\Sigma)$, called $\theta$-line bundle, whose transition functions are defined by

$$
\theta\left[\begin{array}{l}
\alpha \\
\beta
\end{array}\right](z+n+\Omega m)=\exp \left(-i \pi m^{t} \Omega m-i 2 \pi m^{t} z+i 2 \pi\left(\alpha^{t} n-\beta^{t} m\right)\right) \theta\left[\begin{array}{l}
\alpha \\
\beta
\end{array}\right](z) .
$$


The Jacobi map $I: \Sigma \rightarrow C^{g}$ is defined by

$$
I(P)=\int_{P_{0}}^{P} \eta, \quad P_{0}, P \in \Sigma,
$$

where $P_{0}$ is an arbitrary reference point on $\Sigma$.

An important property of the Jacobi map is that it maps divisor classes to $J(\Sigma)$ (Abel's theorem), that is, given a divisor $D=\sum_{i=1}^{m} P_{i}-\sum_{i=1}^{n} Q_{i}, I(D)=\sum_{i=1}^{m} I\left(P_{i}\right)$ $-\sum_{i=1}^{n} I\left(Q_{i}\right)$, then $I(D)=I([D]) \bmod \Gamma(\Sigma)$, where $[D]$ is the divisor class defined by the equivalence relation: $D_{1} \approx D_{2}$ if $\left(D_{1}-D_{2}\right)$ is the divisor of a meromorphic function. In the text we denote for compactness $I(D)$ by $D$ itself.

A fundamental theorem in $\theta$-function theory is the Riemann vanishing theorem. It states that the function

$$
F(P)=\theta\left(I(P)-\sum_{i=1}^{g} I\left(P_{i}\right)+I(\Delta)\right)
$$

either vanishes identically or it has exactly $g$ simple zeros in $P=P_{i}, i=1, \ldots, g$. $\Delta$ is the Riemann divisor class defined by

$$
I_{k}(\Delta)=i \pi-i \pi \Omega_{k k}+\sum_{l \neq k} \oint_{a_{1}} \eta_{1}(P) \int_{P_{0}}^{P} \eta_{k} .
$$

As a useful corollary of this theorem, we have

$$
\theta\left(-\sum_{i=1}^{g-1} I\left(P_{i}\right)+I(\Delta)\right)=0, \quad \forall P_{i} \in \Sigma .
$$

The prime form $E(P, Q)$ is a multivalued -1/2-differential without poles in both variables $P$ and $Q$ with a unique simple zero for $Q=P$,

$$
\begin{aligned}
& E(P, Q)=\frac{\theta\left[\begin{array}{l}
\alpha \\
\beta
\end{array}\right](I(P)-I(Q))}{h(P) h(Q)}=-E(Q, P), \\
& E(P, Q) \approx P-Q, \text { as } Q \approx P,
\end{aligned}
$$

where $h^{2}(P)=\sum_{i=1}^{g} \partial_{z_{i}} \theta\left[\begin{array}{l}\alpha \\ \beta\end{array}\right](0) \eta_{i}(P) . h(P)$ is the holomorphic section of the spin bundle corresponding to a non-singular $\left(\partial_{z_{l}} \theta\left[\begin{array}{l}\alpha \\ \beta\end{array}\right](0) \neq 0\right)$ odd spin structure $\left[\begin{array}{l}\alpha \\ \beta\end{array}\right] \cdot{ }^{3} E(P, Q)$ is independent of the particular choice of $\left[\begin{array}{l}\alpha \\ \beta\end{array}\right]$. For cycles winding around $P$, it transforms as

$$
E(P, Q) \stackrel{P+n a+m b}{\longrightarrow} \exp \left(-i \pi m^{t} \Omega m-i 2 \pi m^{t}(I(P)-I(Q))\right) E(P, Q) .
$$

\footnotetext{
${ }^{3}$ The correspondence between spin bundles and theta characteristics is illustrated in Appendix B
} 
Finally, we introduce the $\sigma$-differential

$$
\sigma(P)=\exp \left(-\sum_{j=1}^{g} \oint_{a_{j}} \eta_{j}(Q) \ln E(Q, P)\right) .
$$

It is a $g / 2$-differential defined on a covering of $\Sigma$ without zeroes and poles. Its transformation property is

$$
\sigma(P) \stackrel{P+n a+m b}{\longrightarrow} \exp \left(i \pi(g-1) m^{t} \Omega m-i 2 \pi m^{t}(I(\Delta)-(g-1) I(P))\right) \sigma(P) .
$$

\section{Appendix B}

In this appendix we illustrate some useful properties of the $\Theta$-divisor [24, 25]. Let $\kappa$ be a degree $(g-1)$ line bundle. It has a holomorphic section if in its corresponding divisor class $[\kappa]$ there is a positive divisor $\sum_{i=1}^{g-1} P_{i}$. By the Riemann-Roch theorem such a divisor exists if and only if $K \otimes \kappa^{-1}$ has a divisor of the form $\sum_{i=1}^{g-1} Q_{i}$. Let $^{4} D_{(\alpha \beta)}$ be a spin bundle (i.e., $2\left[D_{(\alpha \beta)}\right]=[K]$ ), then the set

$$
S_{(\alpha \beta)}=\left\{I\left(\sum_{i=1}^{g-1} P_{i}-D_{(\alpha \beta)}\right) \mid P_{1}, \ldots, P_{g-1} \in \Sigma\right\} \subset J(\Sigma)
$$

is a symmetric subset with respect to the origin of $J(\Sigma)$, that is

$$
-I\left(\sum_{i=1}^{g-1} P_{i}-D_{(\alpha \beta)}\right)=I\left(\sum_{i=1}^{g-1} Q_{i}-D_{(\alpha \beta)}\right) \in S_{(\alpha \beta)} .
$$

From the Riemann vanishing theorem as the points $P_{i}$ sweep $\Sigma$ we recover $\Theta$

Therefore

$$
I(\Delta)-\left\{I\left(\sum_{i=1}^{g-1} P_{i}\right) \mid P_{1}, \ldots, P_{g-1} \in \Sigma\right\}=\Theta .
$$

$$
S_{(\alpha \beta)}=\gamma_{(\alpha \beta)}-\Theta, \quad \gamma_{(\alpha \beta)}=I\left(\Delta-D_{(\alpha \beta)}\right) .
$$

Since $\Theta$ and $S_{(\alpha \beta)}$ are both symmetric subsets with respect to the origin of $J(\Sigma)$, we have

$$
\Theta+2 \gamma_{(\alpha \beta)}=\Theta
$$

This means that $\theta\left(z+2 \gamma_{(\alpha \beta)}\right) / \theta(z)$ is a constant on the compact space $J(\Sigma)$, therefore $2 \gamma_{(\alpha \beta)} \in \Gamma(\Sigma)$, that is each $\gamma_{(\alpha \beta)}$ is one of the $2^{2 g}$ points of order two. Being $2^{2 g}$ also the number of spin structures, it follows from Abel's theorem that for each half-points of $J(\Sigma)$ there is a different $D_{(\alpha \beta)}$ and viceversa. Since we can write $\gamma_{(\alpha \beta)}=\beta+\Omega \alpha$, then

$$
\begin{aligned}
\Theta_{(\alpha \beta)} & \equiv\left\{z: \theta\left[\begin{array}{l}
\alpha \\
\beta
\end{array}\right](z)=0\right\}=\Theta+\gamma_{(\alpha \beta)}, \quad \alpha_{i}, \beta_{i} \in\{0,1 / 2\}, \\
I\left(D_{(\alpha \beta)}\right) & =I(\Delta)-\beta-\Omega \alpha .
\end{aligned}
$$

\footnotetext{
${ }^{4}$ In the following we denote by $D_{(\alpha \beta)}$ both the spin bundle and the divisor class $\left[D_{(\alpha \beta)}\right]$
} 
Noting that

$$
\left\{I\left(\sum_{i=1}^{g-1} P_{i}-D_{(00)}\right) \mid P_{1}, \ldots, P_{g-1} \in \Sigma\right\}=\Theta,
$$

and that $\Theta$ is $P_{0}$-independent we see that $D_{(00)}$ depends only on the homology basis chosen. From (B.4) it follows that there is a one-to-one correspondence between degree $(g-1)$ line bundles for which $\bar{\partial}_{\kappa}$ has a zero mode (that is, the line bundles $\left.\left\{I\left(\sum_{i=1}^{g-1} P_{i}\right) \mid P_{1}, \ldots, P_{g-1} \in \Sigma\right\}\right)$ and points in $\Theta$. Moreover, it turns out that $h^{0}(\Sigma, \kappa)$ equals the multiplicity of the zero of $\theta(z)$ at $z=I(\Delta)-I(\kappa)$.

For odd characteristics $\left[\begin{array}{l}\alpha \\ \beta\end{array}\right], 0 \in \Theta_{(\alpha \beta)}$, so that there is at least a set of points
such that

$$
I\left(\sum_{i=1}^{g-1} P_{i}-D_{(\alpha \beta)}\right)=0,
$$

that is, $D_{(\alpha \beta)}$ has at least a holomorphic section with zeroes at $z=P_{i}, i=1, \ldots, g-1$. In the case of even theta functions there are certain values of $\Omega$ for which $0 \in \Theta_{(\alpha \beta)}$, for example for $g=2$ this happens when the period matrix is diagonal.

Acknowledgements. We would like to thank G. Falqui, J. D. Fay, F. Ferrari, R. Iengo, M. Martellini, C. Reina, M. Rinaldi, and V. Tapia for discussions and advice.

\section{References}

1. Date, E., Jimbo, M., Kashiwara, M., Miwa, T.: In: Proc. of International Symposium on NonLinear Integrable Systems, Kyoto 1981. Jimbo, M., Miwa, T. (eds.). Singapore (1983)

2. Saito, S.: Phys. Rev. Lett. D 36, 1819 (1987); 59, 1798 (1987), and Tokyo Metropolitan University preprints TMUP-HEL-8613/8615

3. Ishibashi, N., Matsuo, Y., Ooguri, H.: Mod. Phys. Lett. A 2, 119 (1987)

Matsuo, Y., preprint UT-511 (1986)

4. Alvarez-Gaumé, L., Gomez, C., Reina, C.: In Proc. of the Trieste Spring School on Superstrings, CERN-TH 4775/87

Alvarez-Gaumé, L., Gomez, C., Moore, G., Vafa, C.: Nucl. Phys. B 303, 455 (1988)

5. Witten, E.: Commun. Math. Phys. 113, 529 (1988)

6. Vafa, C.: Phys. Lett. 190B, 47 (1986); 189, 195 (1987)

7. Kawamoto, N., Namikawa, Y., Tsuchiya, A., Yamada, Y.: Commun. Math. Phys. 116, 247 (1988)

8. Sato, M., Sato, Y.:In: Non-linear partial differential equations in applied science (Tokyo 1982) Fujita, H., Lar, P., Strang, G. (eds.). Amsterdam: North-Holland 1983

9. Segal, G., Wilson, G.: Publ. Math. del I.H.E.S. 61, 1 (1985).

Pressley, A., Segal, G.: Loop groups. Oxford: Oxford University Press 1986

10. Beilinson, A., Manin, Y., Schechtman, Y.A.: Localization of Virasoro and Neveu-Schwarz algebras. Preprint (1986)

Kontsevich, M.: Funct. Anal. Appl. 21, 156 (1987)

11. Arbarello, E., de Concini, C., Kac, V., Procesi, C.: Moduli space of curves and representation theory. Commun. Math. Phys. 117, 1-36 (1988)

12. Krichever, I.M., Novikov, S.P.: Funk. Anal. i Pril 21, 46 (1987) 
13. Krichever, I.M., Novikov, S.P.: Funk. Anal. i Pril 21, 47 (1987)

14. Belavin, A.A., Polyakov, A.M., Zamolodchikov, A.B.: Nucl. Phys. B 241, 333 (1984)

15. Friedan, D., Martinec, E., Shenker, S.: Nucl. Phys. B 271, 93 (1986)

16. Bonora, L., Bregola, M., Cotta-Ramusino, P., Martellini, M.: Phys. Lett. B 205, 53 (1988)

17. Bonora, L., Martellini, M., Rinaldi, M., Russo, J.: Phys. Lett. B 206, 444 (1988)

18. Bonora, L., Rinaldi, M., Russo, J., Wu, K.: Phys. Lett. B 208, 440 (1988)

19. Farkas, H., Kra, I.: Riemann surfaces. Berlin, Heidelberg, New York: Springer 1980

20. Espriu, D.: Phys. Lett. 198B, 171 (1987)

Martellini, M., Sanchez, N.: Phys. Lett. 192B, 361 (1987)

21. Here are a few references on correlation functions in $b-c$ systems: Eguchi, T., Ooguri, H.: Phys. Lett. B 187, 127 (1987)

Atick, J.J., Sen, A.: Phys. Lett. 186B, 339 (1987)

Bonini, M., Iengo, R.: Int. J. Mod. Phys. A 3, 841 (1988)

Sonoda, H.: Phys. Lett. 178B, 390 (1986)

Namazie, M.A., Narain, K.S., Sarmadi, M.H.: Phys. Lett. 177B, 329 (1986)

Knizhnik, V.G.: Phys. Lett. 180B, 247 (1986)

Dugan, M., Sonoda, H.: Nucl. Phys. B 289, 227 (1987)

22. Verlinde, E., Verlinde, H.: Nucl. Phys. B 288, 357 (1987)

23. Schiffer, M., Spencer, D.C.: Functionals on finite Riemann surfaces. Princeton, NJ: Princeton University Press 1954

24. Fay, J.: Theta functions on Riemann surfaces. Lecture Notes in Mathematics, Vol. 356. Berlin, Heidelberg, New York: Springer 1973

Mumford, D.: Tata lectures on theta I and II. Basel: Birkhäuser 1983

25. Alvarez-Gaumé, L., Nelson, P.: Riemann surfaces and string theories. CERN-TH 4615/86

Communicated by G. Parisi

Received June 10, 1988 